\title{
Sound in Human-Robot Interaction
}

Hannah Pelikan, Frederic Anthony Robinson, Leelo Keevallik, Mari Velonaki, Mathias Broth and Oliver Bown

The self-archived postprint version of this conference article is available at Linköping University Institutional Repository (DiVA):

http:/ / urn.kb.se/ resolve?urn=urn:nbn:se:liu:diva-178306

N.B.: When citing this work, cite the original publication.

Pelikan, H., Robinson, F. A., Keevallik, L., Velonaki, M., Broth, M., Bown, O., (2021), Sound in Human-Robot Interaction, HRI '21 Companion: Companion of the 2021 ACM/ IEEE International Conference on Human-Robot Interaction, 706-708. https:// doi.org/ 10.1145/3434074.3444871

Original publication available at:

https:/ / doi.org/ 10.1145/3434074.3444871

Copyright: Association for Computing Machinery (ACM)

http:/ / www.acm.org/

(C) ACM 2021. This is the author's version of the work. It is posted here for your personal use. Not for redistribution. 


\section{Sound in Human-Robot Interaction}

\author{
Hannah Pelikan \\ Linköping University \\ Linköping, Sweden \\ hannah.pelikan@liu.se
}

\author{
Mari Velonaki \\ Creative Robotics Lab \\ University of New South Wales \\ Sydney, Australia \\ mari.velonaki@unsw.edu.au
}

\author{
Frederic Anthony Robinson \\ Creative Robotics Lab \\ University of New South Wales \\ Sydney, Australia \\ frederic.robinson@unsw.edu.au \\ Mathias Broth \\ Linköping University \\ Linköping, Sweden \\ mathias.broth@liu.se
}

\author{
Leelo Keevallik \\ Linköping University \\ Linköping, Sweden \\ leelo.keevallik@liu.se
}

\author{
Oliver Bown \\ Interactive Media Lab \\ University of New South Wales \\ Sydney, Australia \\ o.bown@unsw.edu.au
}

\begin{abstract}
Robot sound spans a wide continuum, from subtle motor hums, through music, bleeps and bloops, to human-inspired vocalizations, and can be an important means of communication for robotic agents. This first workshop on sound in HRI aims to bring together interdisciplinary perspectives on sound, including design, conversation analysis, (computational) linguistics, music, engineering and psychology. The goal of the workshop is to stimulate interdisciplinary exchange and to form a more coherent overview of perspectives on how sound can facilitate human-robot interaction. During the half-day workshop, we will explore (1) the diverse application opportunities of sound in human-robot interaction, (2) strategies for designing sonic human-robot interactions, and (3) methodologies for the evaluation of robot sound. Workshop outcomes will be documented on a dedicated website and are planned to be collected in a special issue.
\end{abstract}

\section{CCS CONCEPTS}

- Human-centered computing $\rightarrow$ Human computer interaction (HCI); Auditory feedback.

\section{KEYWORDS}

human-robot interaction; robot sound; sonic interaction design; robotic musicianship; semantic-free utterances; non-lexical sounds

\section{ACM Reference Format:}

Hannah Pelikan, Frederic Anthony Robinson, Leelo Keevallik, Mari Velonaki, Mathias Broth, and Oliver Bown. 2021. Sound in Human-Robot Interaction. In Companion of the 2021 ACM/IEEE International Conference on HumanRobot Interaction (HRI '21 Companion), March 8-11, 2021, Boulder, CO, USA. ACM, New York, NY, USA, 3 pages. https://doi.org/10.1145/3434074.3444871

\section{INTRODUCTION}

As robots move from laboratories and exhibitions into social environments, nuanced human-robot interaction becomes of growing

Permission to make digital or hard copies of part or all of this work for personal or classroom use is granted without fee provided that copies are not made or distributed for profit or commercial advantage and that copies bear this notice and the full citation on the first page. Copyrights for third-party components of this work must be honored For all other uses, contact the owner/author(s).

HRI '21 Companion, March 8-11, 2021, Boulder, CO, USA

(c) 2021 Copyright held by the owner/author(s).

ACM ISBN 978-1-4503-8290-8/21/03.

https://doi.org/10.1145/3434074.3444871 importance. While most communication in HRI happens in the form of visualizations and speech, sound as a nonverbal resource starts to gain attention as well. Motor sound can influence our impressions and expectations of a robot [3]. Music enables robots to engage and communicate [4] and shared musical experiences foster robot-human relationships [1]. Utterances such as non-linguistic beeps, musical vocalizations, gibberish speech, backchannels (e.g. 'oops', 'aha') and paralinguistic aspects such as intonation patterns have been used to communicate affect and intent [5]. Commercial robots like Cozmo rely heavily on non-lexical audio when interacting with humans [2]. While first steps have been taken to explore robot sounds from a variety of perspectives, there seems to be little dialogue between the different approaches.

\section{WORKSHOP OBJECTIVES}

This workshop aims to provide insight into how sound can be used to improve human-robot interactions by bringing together researchers and speakers from various disciplines in academia and industry. The workshop presents methodologies relevant to ideation, creation and evaluation of sound for HRI. It offers a platform to connect researchers working with sound and those interested in doing so, stimulating discussion and sharing ideas to identify future challenges and opportunities. By exploring the various ways in which sound informs, influences, and engages people, the workshop aims to find new ways of using this modality to create richer, and more nuanced human-robot interactions. Topics include, but are not limited to:

- Methodological approaches to ideation, creation, and evaluation of sound in HRI

- Conceptual contributions on how sound can facilitate new and engaging forms of human-robot interactions

- Empirical studies on the effects of sound in HRI in laboratory and real-world contexts

- Sonic interaction design perspectives on sound in HRI

- Novel applications of sound and music in HRI

- Technologies, systems, toolkits for sound in HRI

- Human-inspired vocalizations for robots 


\section{FORMAT AND SCHEDULE}

The workshop will be held as a half-day online event on Zoom.

Workshop participants will briefly present position papers. As we welcome participants from all kinds of backgrounds, we hope to cover a broad range of perspectives on sound in HRI. Depending on the submissions, we will explore approaches to sound generation, evaluation methods and theoretical issues around how and why sound can facilitate new and interesting "bold" forms of human-robot interaction. The submissions by participants will be distributed across three themed sessions, each of which is preceded by a keynote to frame the discussion. The keynote speakers bring together perspectives from industry and academia, presenting insights from their work on using sound and music to create rich and engaging interactions between humans and robotic agents. The workshop will end with discussion in breakout rooms and a final panel discussion.

Invited speakers: 1) Ben Galbadon, currently interaction sound designer at Facebook. He will present his work on robots Cozmo and Vector, who arguably feature some of the most elaborate sound design currently existing in commercial robots. 2) Guy Hoffman, assistant professor at the Sibley School of Mechanical and Aerospace Engineering at Cornell University. He will discuss his research into anticipation and timing in HRI, musical performance robots, robot improvisation, and nonverbal communication in HRI.

\section{Session I - Ideation}

What are the challenges and opportunities around the use of sound in human-robot interaction? Which of the many forms of sonic expression are applicable in HRI? Which other fields can inform these sonic interactions?

Invited Speaker: Guy Hoffman

Assistant Professor

Cornell University

\section{Session II - Creation}

How can sound in HRI be created and implemented? What are the conceptual and creative implications of designing non-verbal sonic interaction?

Invited Speaker: Ben Gabaldon

Interaction Sound Designer

Facebook, formely Anki

\section{Session III - Evaluation}

What methodologies are effective and appropriate for the evaluation of sonic human-robot interactions? How do we create reproducible findings that can inform future research?

\section{TARGET AUDIENCE}

We welcome researchers and practitioners with an interest in sound for human-robot interaction from a broad variety of backgrounds, including sound design, engineering, conversation analysis \& ethnomethodology, linguistics and psychology.

Submissions should be no longer than 2 pages excluding references and conform to the ACM SIG format. Templates for Word,
Latex, and Overleaf are linked on the workshop website. Submissions do not need to be anonymised and should be sent to frederic.robinson@unsw.edu.au until February 8th. The call for participation for this workshop will be distributed via HRI- and HCIrelated mailing lists (e.g. robotics worldwide and CHI-announcements).

\section{ORGANIZERS}

Hannah Pelikan is a $\mathrm{PhD}$ student in language and culture at Linköping University. Her work combines human-robot interaction and multimodal conversation analysis and explores how sound can facilitate coordination between robot and human bodies. Hannah studies robot sounds through video recordings of robots in realworld settings, such as in the home or in regular traffic.

Frederic Robinson is a Scientia $\mathrm{PhD}$ Candidate at the University of New South Wales' Creative Robotics Lab \& Interactive Media Lab. His research investigates the potential of spatial sonic interaction design to create richer and more engaging human-robot interactions, informed by a background in interactive multi-channel sound for large-scale media installations.

Leelo Keevallik is a professor in language and interaction at Linköping University. She has worked on the interface between grammar and embodiment, targeting the systematic use of language, sound, and movement in various activities involving co-present humans. Among other things, she has studied how vocalizations are used to coordinate manual work tasks and dance instruction.

Mari Velonaki is director of the UNSW Creative Robotics Lab and lead chief investigator at the National Facility for Human Robot Interaction. She has worked as an artist and researcher in the field of electronic art since 1997 pioneering intellectually and emotionally engaging human-machine interfaces through the creation of multisensory experiences. Her robots and interactive installations have been exhibited worldwide, including: V\&A Museum, London; National Art Museum Beijing; Aros Aarhus Museum of Modern Art, Denmark; Ars Electronica, Linz; and ZENDAI Museum of Modern Art, Shanghai.

Mathias Broth is professor in language and interaction at Linköping University. Taking particular interest in the embodied, emplaced and mediated aspects of situated human interaction, he has studied activities such as TV-production and driver training as these are interactionally accomplished in real time.

Oliver Bown is a senior lecturer at the UNSW Faculty of Art and Design. As a researcher and maker working with creative technologies, he comes from a highly diverse academic background spanning social anthropology, evolutionary and adaptive systems, music informatics and interaction design. His current active research areas include media multiplicities, musical metacreation, the theories and methodologies of computational creativity, new interfaces for musical expression, and multi-agent models of social creativity.

\section{DOCUMENTATION}

After the workshop, participants will be given the opportunity to extend their position papers, combining their initial submission with insights obtained during the workshop. Participants with aligned interests are encouraged to collaborate on submissions. Final submissions will be collected and reviewed by the organising 
committee, which plans to formally publish workshop submissions as a special issue on sound in HRI through ACM. Key insights from the workshop will be made available on the workshop's website, https://robinson.audio/SoundInHRI.

\section{REFERENCES}

[1] Guy Hoffman, Shira Bauman, and Keinan Vanunu. 2016. Robotic experience companionship in music listening and video watching. Personal and Ubiquitous Computing 20, 1 (Feb. 2016), 51-63. https://doi.org/10.1007/s00779-015-0897-1

[2] Hannah R. M. Pelikan, Mathias Broth, and Leelo Keevallik. 2020. "Are You Sad, Cozmo?": How Humans Make Sense of a Home Robot's Emotion Displays. In Proceedings of the 2020 ACM/IEEE International Conference on Human-Robot Interaction
(Cambridge, United Kingdom) (HRI '20). Association for Computing Machinery, New York, NY, USA, 461-470. https://doi.org/10.1145/3319502.3374814

[3] Hamish Tennent, Dylan Moore, Malte Jung, and Wendy Ju. 2017. Good vibrations: How consequential sounds affect perception of robotic arms. In 2017 26th IEEE International Symposium on Robot and Human Interactive Communication (ROMAN). IEEE, Lisbon, 928-935. https://doi.org/10.1109/ROMAN.2017.8172414

[4] Gil Weinberg, Mason Bretan, Guy Hoffman, and Scott Driscoll. 2020. Robotic Musicianship: Embodied Artificial Creativity and Mechatronic Musical Expression. Vol. 8. Springer Nature.

[5] Selma Yilmazyildiz, Robin Read, Tony Belpeame, and Werner Verhelst. 2016. Review of semantic-free utterances in social human-robot interaction. International Fournal of Human-Computer Interaction 32, 1 (Jan. 2016), 63-85. https: //doi.org/10.1080/10447318.2015.1093856 